\title{
Three-dimensional Trajectory Tracking System Based on Compass and
}

\section{Gyroscope}

\author{
Jia Ming Deng ${ }^{1, a^{*}}$, Jun Shuai Qiü, b , Zhi Yuan Zhong ${ }^{3, c}$ and Zhi Ping Wan ${ }^{4, d}$ \\ 1,2,3,4 Xinhua College of Sun Yat-sen University, Guangzhou 510520, China

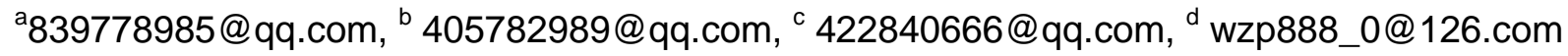

Keywords: Beidou navigation; Embedded; Three-dimensional space; Trajectory tracking; Location

\begin{abstract}
According to the high-precision positioning capability with Beidou navigation and the angular momentum conservation of gyroscope, this paper presents a real-time trajectory tracking system based on embedded wireless three-dimensional space. The system used by the application which based QT-cross-platform C ++ graphical user interface. And it is used the OpenGL to achieve real-time trajectory record and precise positioning. And data integration use of Qlite database technology. Experimental results show that simulation results of this system basically consistent with expected results. It has advantages of the long positioning and strong spatial expression.
\end{abstract}

\section{Introduction}

Compass/Beidou Navigation Satellite System is being implemented by the independent development and independent global satellite navigation system. In 2012, it is the ability to cover the Asia-Pacific region positioning, navigation and timing, short-message communication service at first [1]. And will be in 2020, it will be completion the Beidou satellite navigation system which cans global coverage. After the completion of the Beidou system, it will provide users with positioning, timing and packet communications integration service [2]. And Beidou system is compatible with other satellite navigation systems. That is allows users to simultaneously use multiple systems observed data [3-4]. It is greatly improved observation redundancy, improve navigation and positioning accuracy.

On this basis, this paper presents a trajectory tracking systems that based on three-dimensional space. Systems to achieve are based on MEMS strap down navigation system. There in, MEMS strap down navigation system is using the gyroscope to measurement Spatial Data of the vector. In the last, we can get the athletic stance, velocity and trajectory. At the same time, this paper is combined with Compass/GPS navigation system that can improve the accuracy of the data.

\section{The overall structure of the Compass/GPS navigation}

Beidou satellite navigation system consists of three parts, such as the space side, the ground and the clients.it can provide services for all types of users across the globe around the clock and all-time. And it has the ability to high precision and high reliability positioning, short packets in communication. Nowadays, China has initially with area navigation, positioning and timing capabilities. The accuracy of the data meet the following data, Positioning accuracy of 10 meters, velocity accuracy of $0.2 \mathrm{~m} / \mathrm{s}$, timing precision is 10-nanosecond. Ground terminal is made up of the master station, injection stations and several ground stations. The client is made up of various types 
of receivers and the signal processor. Close coordination between the three together forms the Beidou navigation architecture receiver module [5-6].

\section{Hardware Design}

Hardware circuit. Circuit is mainly design by integrated gyroscope module of L3G4200 and accelerometer module of ADXL345 integration [7-8].
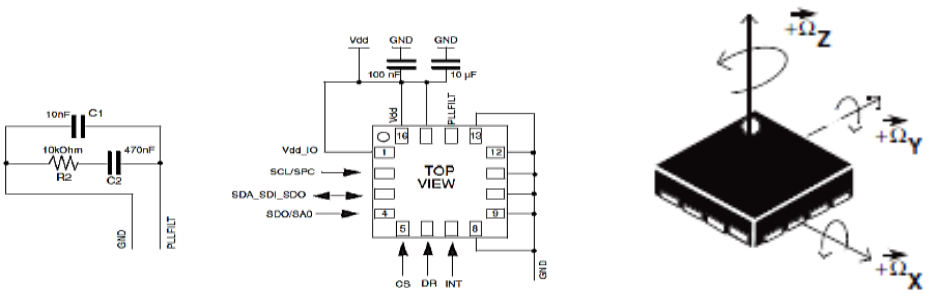

Figure. 1 Schematic diagram L3G4200 module

Power supply decoupling capacitors (100 $\mathrm{nF}$ ceramic or polyester $+10 \mu \mathrm{F})$ should be placed as near as possible to the device (common design practice). If Vdd and Vdd_IO are not connected together, power supply decoupling capacitors ( $100 \mathrm{nF}$ and $10 \mu \mathrm{F}$ between Vdd and common ground, $100 \mathrm{nF}$ between Vdd_IO and common ground) should be placed as near as possible to the device (common design practice). The L3G4200 IC includes a PLL (phase locked loop) circuit to synchronize driving and sensing interfaces. Capacitors and resistors must be added at the PLLFILT pin (as shown in Figure 2) to implement a second-order low-pass filter.

Selection of MPU6050. The system uses a six-axis module MPU6050, it combines a high-precision gyroscopes and accelerometers. It is read the measurement data through the processor MPU6050, and through serial output the data. That is eliminating the need for users to develop their own MPU6050 complex I2C protocol. It also has a strong anti-jamming capability, the measurement accuracy.

The MPU-60X 0 Motion Tracking devices, with its 6-axis integration, on-board Motion Fusion ${ }^{\mathrm{TM}}$, and run-time calibration firmware, enables manufacturers to eliminate the costly and complex selection, qualification, and system level integration of discrete devices, guaranteeing optimal motion performance for consumers. The MPU-60X0 is also designed to interface with multiple non-inertial digital sensors, such as pressure sensors, on its auxiliary I2 C port. The MPU-60X0 is footprint compatible with the MPU-30X 0 families.

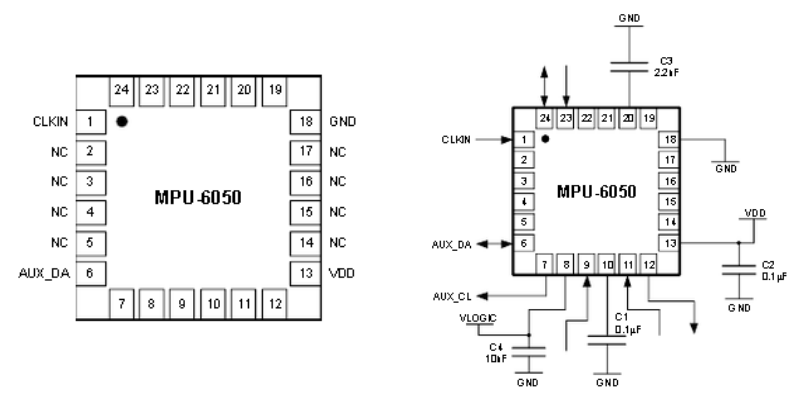

Figure. 2 Chart of MPU6050

The MPU-60X0 features three 16-bit analog-to-digital converters (ADCs) for digitizing the gyroscope outputs and three 16-bit ADCs for digitizing the accelerometer outputs. For precision tracking of both fast and slow motions, the parts feature a user-programmable gyroscope full-scale 
range of $\pm 250, \pm 500, \pm 1000$, and $\pm 2000 \%$ sec (dps) and a user-programmable accelerometer full-scale range of $\pm 2 \mathrm{~g}, \pm 4 \mathrm{~g}, \pm 8 \mathrm{~g}$, and $\pm 16 \mathrm{~g}$.

Selection Module. The system uses Compass/GPS dual-mode module is BD-288 models. It is high-performance module support Beidou and GPS. And it has a full range of capabilities of satellite positioning receiver. Not only to meet the stringent requirements of professional orientation, but also able to meet the experience needs of the individual consumer. The product has been used in the development of a number of areas. Such as automotive safety systems, vehicle navigation and vehicle monitoring, etc.

\section{Software Design}

Acceleration. Based on the principle of mathematical physics, By calculating the acceleration double integral of the moving object, we can get motion displacement of objects. In the tracking module, we use high-frequency acceleration sensor continuously checks the destination. At the same time, acceleration data of the three groups was integrated to get the space trajectory. Method is as follows.

Assumed to begin sampling from t0, this paper is according to the principle of integration. We can obtain the following functional [9].

$$
S(t)=\int_{t_{0}}^{t} v(t) d t+s\left(t_{0}\right) ; \quad v(t)=\int_{t_{0}}^{t} a(t) d t+v\left(t_{0}\right)
$$

Therein, $\mathrm{s}(\mathrm{t} 0)$ is the cumulative displacement from 0 to $\mathrm{t} 0 . \mathrm{v}(\mathrm{t} 0)$ is the instantaneous velocity of the system at time t0.Make it satisfies the initial conditions $\mathrm{s}(\mathrm{t} 0)=0$, Get functional as follows.

$$
S(t)=\int_{t_{0}}^{t} V(t) d t=\frac{V\left(t_{0}\right)+V\left(t_{1}\right)}{2}\left(t_{1}-t_{0}\right)+\frac{V\left(t_{2}\right)+V\left(t_{1}\right)}{2}\left(t_{2}-t_{1}\right)+\cdots+\frac{V\left(t_{n}\right)+V\left(t_{n-1}\right)}{2}\left(t_{n}-t_{n-1}\right)
$$

Due to $\mathrm{t} 1-\mathrm{t} 0=\mathrm{t} 2-\mathrm{t} 1=\ldots=\mathrm{tn}-\mathrm{tn}-1=\Delta \mathrm{t}$, therein $\Delta \mathrm{t}$ is an acceleration sensor interval.

$$
s(t)=\sum_{k=1}^{n} \frac{v\left(t_{k}\right)+v\left(t_{k-1}\right)}{2} \Delta t
$$

In the discrete domain is rewritten as: $s[n]=\sum_{k=1}^{n} \frac{v(k)+v(k-1)}{2} \Delta t$

When $\mathrm{n}>1$, Discrete domains satisfy the following function.

$$
\begin{aligned}
& v[n]=\sum_{k=1}^{n} \frac{a(k)+a(k-1)}{2} \Delta t=v[0]+\frac{1}{2}(a[0]+a[n]) \cdot \Delta t+(a[1]+a[2]+\cdots+a[n-1]) \cdot \Delta t \\
& s[n]=\sum_{k=1}^{n} \frac{v(k)+v(k-1)}{2} \Delta t=\frac{1}{2}(v[0]+v[n]) \cdot \Delta t+(v[1]+v[2]+\cdots+v[n-1]) \cdot \Delta t
\end{aligned}
$$

Thus we can be obtained the uniaxial moving distance as follows.

$$
s[n]=n \cdot v[0] \cdot \Delta t+[(n-1) \cdot a[1]+(n-2) \cdot a[2]+\cdots+v[n-1]] \cdot \Delta t^{2}+\frac{1}{4}(a[0]+a[n]) \cdot \Delta t^{2}
$$

Eliminate gravity component. Because the process of target tracking space objects will appear flipped, the acceleration of gravity caused by the offset component in the acceleration sensor X, Y, Z-axis. Therefore, we should eliminate the acceleration component of gravity [10]. 


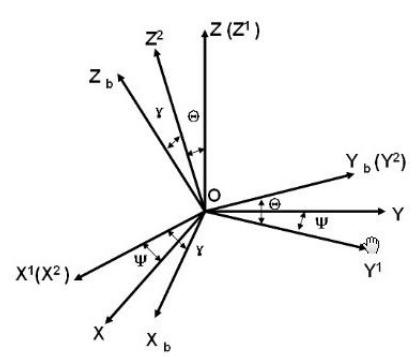

Figure. 3 Coordinate frames

According to Euler angles theorem, we can get attitude transformation matrix.

$\left[\begin{array}{l}x_{b} \\ y_{b} \\ z_{b}\end{array}\right]=\left[\begin{array}{ccc}\cos \gamma & 0 & -\sin \gamma \\ 0 & 1 & 0 \\ \sin \gamma & 0 & \cos \gamma\end{array}\right]\left[\begin{array}{ccc}1 & 0 & 0 \\ 0 & \cos \theta & \sin \theta \\ 0 & -\sin \theta & \cos \theta\end{array}\right]\left[\begin{array}{ccc}\cos \psi & \sin \psi & 0 \\ -\sin \psi & \cos \psi & 0 \\ 0 & 0 & 1\end{array}\right]\left[\begin{array}{l}x \\ y \\ z\end{array}\right]$
$=\left[\begin{array}{ccc}\cos \gamma \cos \psi+\sin \gamma \sin \theta \sin \psi & -\cos \gamma \sin \psi+\sin \gamma \sin \theta \sin \psi & -\sin \gamma \cos \theta \\ \cos \theta \sin \psi & \cos \theta \cos \psi & \sin \theta \\ \sin \gamma \cos \psi-\cos \gamma \sin \theta \sin \psi & -\sin \gamma \sin \psi-\cos \gamma \sin \theta \sin \psi & \cos \gamma\end{array}\right]\left[\begin{array}{l}x \\ y \\ z\end{array}\right]$

This conversion matrix with gravity on the carrier coordinate offset component.Therefore, As long as the rotation matrix is calculated, we can elimination of gravity component.We calculated the Euler angular velocity, which make the rotation matrix changes with the gyroscope. Thus we can eliminate the effects of gravity at any time.

$\left[\begin{array}{l}g_{x} \\ g_{y} \\ g_{z}\end{array}\right]=\left[\begin{array}{ccc}\cos \gamma \cos \psi+\sin \gamma \sin \theta \sin \psi & \cos \gamma \sin \psi-\sin \gamma \sin \theta \sin \psi & -\sin \gamma \cos \theta \\ -\cos \theta \sin \psi & \cos \theta \cos \psi & \sin \theta \\ \sin \gamma \cos \psi+\cos \gamma \sin \theta \sin \psi & \sin \gamma \sin \psi-\cos \gamma \sin \theta \sin \psi & \cos \gamma \sin \theta\end{array}\right]\left[\begin{array}{l}0 \\ 0 \\ g\end{array}\right]$

\section{Simulation}

In this paper is choice of location for the experiment a school dormitory downstairs space. It is about 100 square meters. Meet the requirements of the experiment because of it is fewer people and larger space. In order to facilitate observation, this paper is design of a simulated environment. It is draw a square three-dimensional space to real-time recording and display the path.

Start test, we let a people walk around and holding the device in this open space. This paper is make two paths tracking test. Observe the following simulation. We can see that system drawing a square like the Figure 4 and Figure 5. At the Figure 4, the student is according to the plan of not go back to the origin. We can see that there is no return to the origin of the three-dimensional software. At the Figure 5, we let the students walking one round. We can see that there is depicts a circular image of the three-dimensional software. Therefore, by experiment it can be concluded which system can accurately draw the route taken by the user.

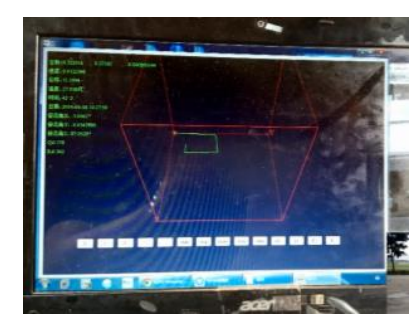

Figure. 4 System drawing a square. Figure. 5 System Drawing a circle. 
While the system tests, the system will generate the corresponding data. As shown below. Through the user's real-time monitoring, system will be timely updates and records. Including the path coordinates, walking speed, displacement, the current temperature. System can be integrating the data to store in the database. In the last, the collected data will be fed back to the client on the phone. And it is easy to observe the user's own location and surroundings.

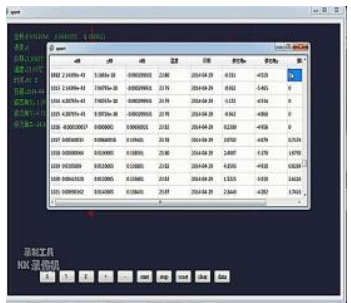

Figure. 6 Database

\section{Conclusion}

This paper presents a wireless three-dimensional trajectory tracking system that is based on Beidou navigation and gyroscope embedded. By accordance with good directional characteristics and positioning features of Beidou navigation and gyroscopes, we proposed navigation system based on MEMS inertial. After the software and hardware design of the system, gets the dimensional space trajectory tracking system which has many advantages, such as low cost, small size, high reliability, and strong anti-jamming capability. It can make up for the problems that unable to locate a long time and unable to provide accurate latitude and longitude.

\section{References}

[1] Bhuiyan MZH, Soderholm S, Thombre S, Ruotsalainen, L, Kuusniemi H. Overcoming the Challenges of BeiDou Receiver Implementation [J]. SENSORS. 2014,14(11):22082-22098.

[2] Jan SSh, Tao AL. The Open Service Signal in Space Navigation Data Comparison of the Global Positioning System and the BeiDou Navigation Satellite System [J]. SENSORS. 2014,14(8): 15182-15202.

[3] Liu JH, Gu DF, Ju B, Yao J, Duan XJ, Yi DY. Basic performance of BeiDou-2 navigation satellite system used in LEO satellites precise orbit determination [J]. CHINESE JOURNAL OF AERONAUTICS. 2014,27(5):1251-1258.

[4] Odolinski R, Odijk D, Teunissen PJG. Combined GPS and BeiDou Instantaneous RTK Positioning [J]. NAVIGATION-JOURNAL OF THE INSTITUTE OF NAVIGATION. 2014,61(2):135-148.

[5] Xu AG, Xu ZQ, Xu XC, etc. Precise Point Positioning Using the Regional BeiDou Navigation Satellite Constellation [J]. JOURNAL OF NAVIGATION. 2014,67(3):523-537.

[6] Nadarajah N, Teunissen PJG, Raziq N. Instantaneous BeiDou-GPS attitude determination: A performance analysis [J]. ADVANCES IN SPACE RESEARCH. 2014,54(5):851-862.

[7] Arai Takeshi, Obuchi Shuichi, Shiba, Yoshitaka, etc. The validity of an assessment of maximum angular velocity of knee extension (KE) using a gyroscope [J]. ARCHIVES OF GERONTOLOGY AND GERIATRICS. 2012,54(2):175-180.

[8] Gataric G, Sipp A, Rowley B, etc. EFFICACY OF A NEW GYROSCOPE-BASED SENSOR 
IN DETECTING PRE-FALL POSTURAL SWAY [J].GERONTOLOGIST. 2008,48(3):633-633

[9] Liu W, Hu Y. Application of generalized binary offset carrier modulation in BeiDou satellite navigation system [J]. JOURNAL OF COMMUNICATIONS TECHNOLOGY AND ELECTRONICS. 2014,59(11):1206-1214.

[10]Cai CS, Pan L, Gao Y. A Precise Weighting Approach with Application to Combined L1/B1 GPS/BeiDou Positioning [J]. JOURNAL OF NAVIGATION. 2014,67(5):911-925. 